\title{
First principle study of the interaction and charge transfer between graphene and organic molecules
}

\author{
Mei Chi, Ya-Pu Zhao* \\ State Key Laboratory of Nonlinear Mechanics, Institute of Mechanics, Chinese Academy of Sciences, Beijing 100190, People's Republic of China
}

\section{A R T I C L E I N F O}

Article history:

Received 15 November 2011

Received in revised form 27 December 2011

Accepted 31 December 2011

Available online 2 February 2012

\section{Keywords:}

First principle study

Graphene

Organic molecules

Adsorption

\begin{abstract}
A B S T R A C T
To search for an effective way to control the carrier type and concentration of graphene, the adsorptions of the electrophilic molecules (TCNE, TCNQ and F4-TCNQ) and the nucleophilic molecules (TDAE and ANTR) on the graphene sheet were investigated using first principle theory calculations. Mulliken population analysis results showed that electrons transferred from the graphene to TCNE, TCNQ and F4-TCNQ, while from TDAE to the graphene sheet. Furthermore, the density of states (DOS) result also was consistent with this. We hope our calculations will be useful for the application of graphene in nanoelectronic devices.
\end{abstract}

(C) 2012 Elsevier B.V. All rights reserved.

\section{Introduction}

Graphene has attracted wide attention since its discovery in 2004 [1] due to its unique structure and properties, it shows numerous potential applications in nanoelectronic devices, such as high speed logic devices, sensors and transparent electrodes [2-7]. Experimental and theoretical researches indicated that graphene has high crystal quality with low density of defects $[8,9]$, in which charge carriers can travel thousands of interatomic distances without being scattered [10-12] and behave as massless relativistic particles. This results in very high electron mobility in graphene, a carrier mobility in excess of $200,000 \mathrm{~cm}^{2} /(\mathrm{V} \mathrm{s})$ was measured for a single layer of mechanically exfoliated graphene [13]. Graphene can be used in ballistic transistor with such high electron mobility [3]. Graphene will bring a major breakthrough in developing future generations of nanoelectronic devices.

However, for further developing of graphene-based devices, controlling the carrier type and concentration of graphene is the most important content. It had been recently shown that $n$-type carrier concentration in epitaxial graphene could be realized by deposited alkali metal atoms on graphene [14]. However, the addition of heteroatoms will destroy the crystal quality and reduce the carrier mobility of graphene. Very recently, Chen et al. [15] obtained p-type doped graphene by adsorbed F4-TCNQ on the graphene surface. They found that electrons transferred from graphene to the F4TCNQ molecule. This result was also confirmed by Lu et al. [16] with

\footnotetext{
* Corresponding author.

E-mail address: yzhao@imech.ac.cn (Y.-P. Zhao).
}

density functional theory (DFT). It provided a nondestructive way for controlling the carrier type and concentration of graphene by adsorbing organic molecules with different electronic properties. So, it is very important to understand the interaction between organic molecules and graphene. However, up to now, there has been few theoretical research on the adsorption of organic molecules on graphene. In this paper, we investigated the adsorption of the electrophilic molecules (TCNE, TCNQ and F4-TCNQ) and the nucleophilic molecules (TDAE and ANTR) on the graphene surface with first principle study. The former is well known as electron acceptor organic molecules with adiabatic electron affinity of 3.17, 2.80 and $3.38 \mathrm{eV}$, respectively. The later is well known as strong donor organic molecules with adiabatic ionization energy of 5.36 and $7.36 \mathrm{eV}$, respectively. We found that both electrophilic and nucleophilic molecules will affect the geometrical and electronic structures of graphene through different ways. And the relationship between the adiabatic electron affinity of electrophilic molecules and the electron transfer was revealed will be useful for the application of graphene in nanoelectronic devices.

\section{Method}

All DFT calculations were performed using DMol ${ }^{3}$ code $[17,18]$ to study the interactions between organic molecules and the graphene surface. Structure optimizations and corresponding total energy calculations of the most stable geometries were based on the generalized gradient approximation (GGA) function with the Perdew-Burke-Ernzerhof (PBE) correction [19]. Compared to local density approximation (LDA), GGA will not lead to a strong 
bonding of molecules. So, if the calculated molecules adsorb on graphene surface, they will definitely bind in a real system $[20,21]$. The DFT semicore pseudopotentials (DSPP) core treatment [22] was implemented for relativistic effects, which replaces core electrons by a single effective potential. To ensure that the results of the calculations were comparable, identical conditions were employed for organic molecules, the graphene substrate and also the adsorbed graphene system. The $k$-point was set to $8 \times 8 \times 1$ for all slabs, which brings out the convergence criterion of $10^{-5}$ hartree on energy and electron density, and that of maximum force of 0.002 hartree/Å. Self-consistent field procedure was carried out with a convergence criterion of $10^{-6}$ hartree on energy and electron density.

To study the adsorption of organic molecules on graphene, a $8 \times 8$ graphene supercell (128 carbon atoms per graphene layer) was used. This was chosen to keep the separate distance between organic molecules large enough to eliminate their interaction in neighboring supercells. Five high-symmetry adsorption sites of organic molecules on graphene were considered in the present study, and they are schematically shown in Fig. 1. Initially, the organic molecule was put with its center on the five sites and with its long axis $(\mathrm{C}=\mathrm{C}$ double bond direction except ANTR) along armchair (sites 1 and 2) or zigzag (sites 3, 4 and 5) direction. Furthermore, the center of organic molecule was above the hollow site of graphene for binding sites 1 and 5 . In contrast, the center of organic molecule was over the bridge site of graphene for sites 2 and 4 . At site 3 , the center of organic molecule was above a carbon atom of graphene.

Full geometry optimization had been performed for all these organic molecules (TCNE, TCNQ F4-TCNQ, TDAE and ANTR) and $8 \times 8$ graphene separately prior to the optimization of the adsorption system. The optimized geometry of the free graphene sheet showed C $-\mathrm{C}$ bond length of $1.42 \AA$, which was in good agreement with previous reports.

\section{Results and discussion}

To find the most favorable adsorption configurations, we first calculated the binding energy $\left(E_{b}\right)$ of adsorbed systems, which is defined as

$E_{b}=E_{O M-G}-\left(E_{G}+E_{O M}\right)$,

where $E_{O M-G}, E_{G}$ and $E_{O M}$ denote the total energy of adsorbed system, isolated graphene and organic molecule, respectively. The most stable configurations of the TCNE, TCNQ F4-TCNQ TDAE and ANTR molecules on the graphene sheet were summarized in Fig. 2. More detailed information for favorable adsorption configurations from the calculation, including values of adsorption energy,

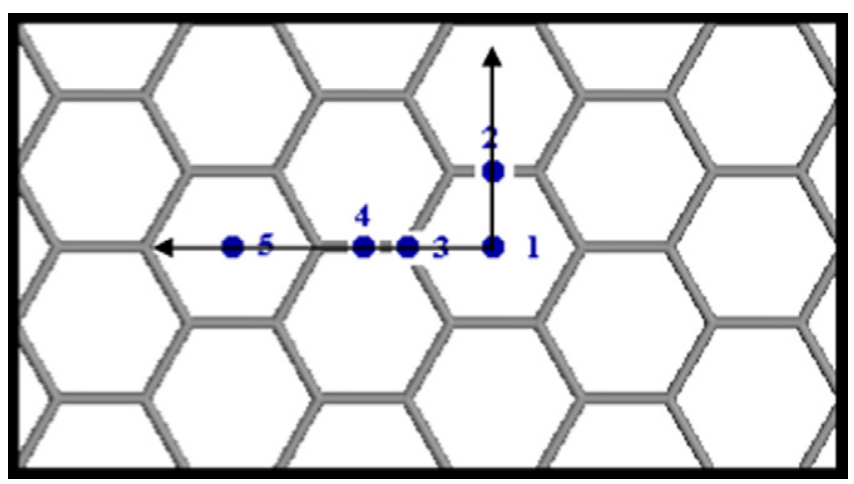

Fig. 1. Schematic top view of five high-symmetry adsorption sites on grapheme. equilibrium graphene-molecule distance (defined as the center -to-center distance of nearest atoms between graphene and organic molecule) and the charge transfer (Mulliken charge), were listed in Table 1 . As we can see, for all organic molecules, $E_{b}$ was negative, which meant that the molecules can be adsorbed on graphene sheet from the thermodynamical viewpoint. From the optimized geometries, we found that for TCNE and ANTR, the structure with its long axis along armchair direction would be energy favorable. In contrast, for TCNQ, F4-TCNQ and TDAE, the configuration with its long axis along zigzag direction would be most stable.

\subsection{TCNE on graphene}

The most stable structure of TCNE on graphene was shown in Fig. 2a. The central $\mathrm{C}=\mathrm{C}$ bond of TCNE was above the hollow site of graphene with its $\mathrm{C}=\mathrm{C}$ bond along the armchair direction. This was in quite agreement with Lu's results [16]. Though structure in Fig. 2a was the most stable adsorption site, the binding energy differences of various sites were no more than $0.03 \mathrm{eV}$. The adsorbed TCNE molecule was found floating above the graphene layer at about 3.4 $\AA$ and no evidence of formation of chemical bonds between them [16]. A charge transfer of $\sim 0.274$ e from graphene to TCNE molecule was found. Almost 0.24 e was transferred to four $\mathrm{C} \equiv \mathrm{N}$ groups while the other transferred to the other two carbon atoms of TCNE, leaving holes in the graphene sheet behind.

\subsection{TCNQ on graphene}

According to the binding energy, the configuration in Fig. 2b represented the energetically most stable configuration with $E_{b}=$ $-0.255 \mathrm{eV}$, and TCNQ-graphene equilibrium distance of $3.497 \AA$. The bezenoid ring of TCNQ was stabilized above the $\mathrm{C}-\mathrm{C}$ bond of the graphene sheet with its long axis along the zigzag direction. This tendency was in agreement with the ab initio results of TCNQ doped $(5,5)$ armchair single walled carbon nanotubes (SWCNTs) [22]. The Mulliken population analysis revealed that the electron charge transfer about 0.26 e occurred from graphene to TCNQ molecule. To better understand the interfacial charge rearrangements between TCNQ and graphene, the deformation charge density $\Delta \rho$ of adsorbed system was calculated, which illustrated how the charge density changes during this adsorption process. $\Delta \rho$ was defined as

$\Delta \rho=\rho_{T}-\left(\rho_{G}+\rho_{\text {OM }}\right)$

in which $\rho_{T}, \rho_{G}$ and $\rho_{O M}$ denoted electron density of the TCNQgraphene adsorbed system, graphene and organic molecule for the adsorbed system, respectively. Contour plots of the charge rearrangements upon adsorption calculated using Eq. (2) were shown in Fig. 3 together with a structure representation of the TCNQ molecular. In Fig. 3a, electrons flowed from the red ${ }^{1}$ areas to the gray areas. Compared with the results present in Fig. $3 \mathrm{~b}$ and Table 2 , the regions of electron accumulation were concentrated on those bonds that were shortened upon adsorption.

\subsection{F4-TCNQ on graphene}

Similar to its precursor TCNQ, in which all four fluorine atoms are replaced by hydrogen atoms, the most stable structure for F4-TCNQ adsorbed system was same as TCNQ adsorbed system. The long axis of F4-TCNQ pointed to the zigzag direction and the bezenoid ring of F4-TCNQ was stabilized above the $\mathrm{C}-\mathrm{C}$ bond of the graphene sheet, with $E_{b}=-0.255 \mathrm{eV}$, and TCNQ-graphene equilibrium distance of 3.497 A. F4-TCNQ was known to adopt a fully

\footnotetext{
${ }^{1}$ For interpretation of color in Figs. 1-5, the reader is referred to the web version of this article.
} 
(a)

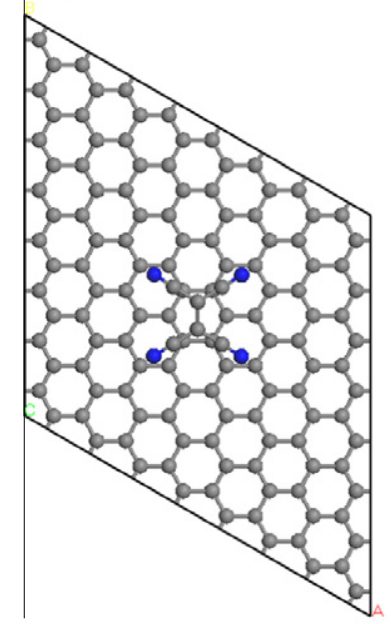

(d)

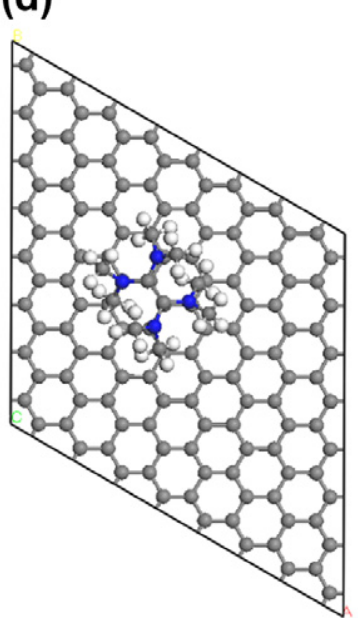

(b)

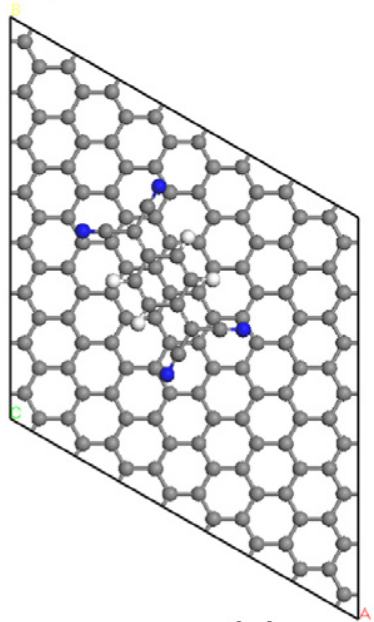

(e) (c)

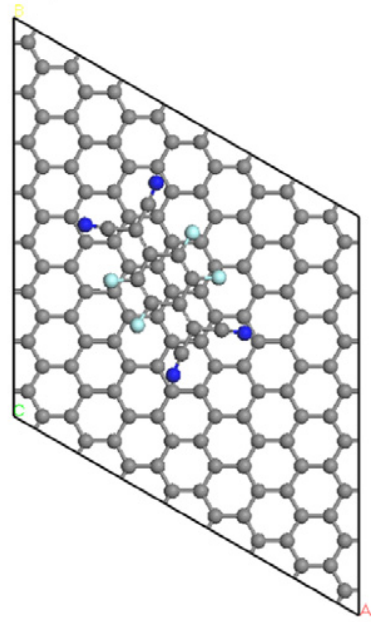

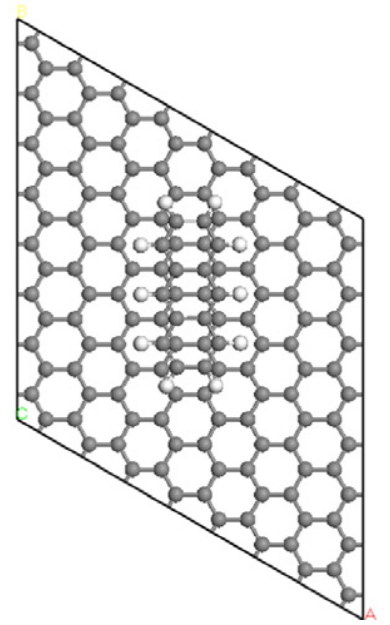

Fig. 2. Most stable configurations of the TCNE (a), TCNQ (b), F4-TCNQ (c), TDAE (d) and ANTR (e) molecules on the graphene sheet.

Table 1

Calculation results of the adsorption energy $E_{b}$, equilibrium graphene-molecule distance $d$ and the charge transfer $q$ for the favorable adsorption configurations.

\begin{tabular}{llll}
\hline Adsorbed system & $E_{b}(\mathrm{eV})$ & $d(\AA)$ & $q(\mathrm{e})$ \\
\hline TCNE + graphene & -0.233 & 3.401 & -0.274 \\
TCNQ + graphene & -0.255 & 3.497 & -0.263 \\
F4TCNQ + graphene & -0.635 & 3.508 & -0.506 \\
TDAE + graphene & -0.417 & 3.082 & 0.454 \\
ANTR + graphene & -0.151 & 3.748 & 0.002 \\
\hline
\end{tabular}

planar and quinoidal structure in the gas phase or as a molecular crystal [23]. This situation changes upon adsorption on graphene: F4-TCNQ adopted a bent geometry with the nitrogen atoms $0.188 \AA$ closer to the graphene sheet than the $\pi$ backbone, which indicated a strong attractive interaction between the $-\mathrm{CN}$ substituents and the graphene sheet. Moreover, a distortion of the molecular skeleton form a quinoidal toward an aromatic structure was observed. This aromatic stabilization could be identified when comparing the bond-length changes in Table 3. The Mulliken population analysis revealed that about 0.51 e charge transformation occurred from graphene to F4-TCNQ molecule. The Mulliken population results also revealed that for the electrophilic molecules, the more negative adiabatic electron affinity is, the more electron transfers to graphene. The structure change and charge transfer results were similar to that of F4-TCNQ adsorption on $\mathrm{Ag}$ (111) surface [24]. Carbon has a similar electron affinity (1.26 eV) as Ag $(1.30 \mathrm{eV})$ [25]. Fig. 4 and Table 3 showed that the regions of electron accumulation are concentrated on those bonds that were shortened upon adsorption.

\subsection{TDAE on graphene}

The energy favorable structure for TDAE adsorbed system was shown in Fig. 2d. The molecular center of TDAE was above the $\mathrm{C}-\mathrm{C}$ bond of graphene and its long axis was along the armchair direction. The adsorbed TDAE molecule was found floating above the graphene layer at about $3.1 \AA$ and no evidence of formation of chemical bonds between them. A charge transfer of $\sim 0.454 \mathrm{e}$ from TDAE molecule to graphene was found.

\subsection{ANTR on graphene}

The energy favorable structure for ANTR adsorbed system was shown in Fig. 2e. The central phenyl ring of ANTR was above the $\mathrm{C}-\mathrm{C}$ bond of graphene and its long axis was along the zigzag direction. The adsorbed ANTR molecule was found floating above the graphene layer at about $3.7 \AA$ and no evidence of formation of chemical bonds between them. A small charge transfer of 
(a)



(b)

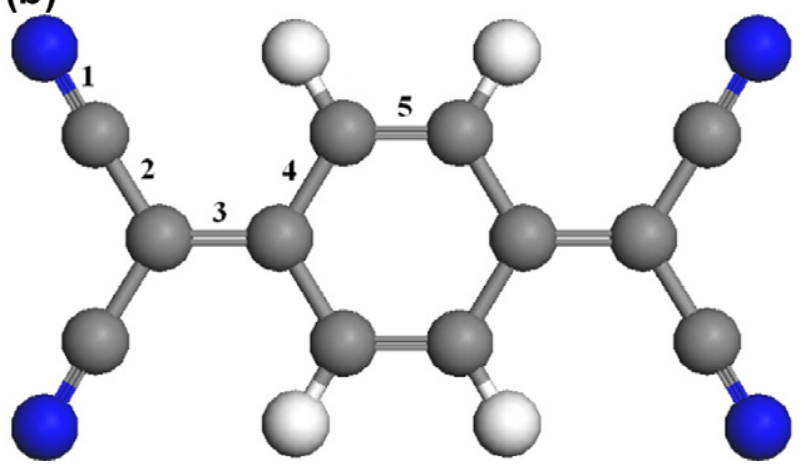

Fig. 3. Electron density difference isosurfaces (a) (the gray region shows the electron accumulation, while the red region shows the electron loss) and a structure representation of the TCNQ molecular (b) (the dark gray, white and blue atoms represent $\mathrm{C}, \mathrm{H}$ and $\mathrm{N}$, respectively) for TCNQ adsorption system.

Table 2

Bond lengths change for TCNQ before and after adsorption (bonds 1-5 are shown in Fig. 3b).

\begin{tabular}{lllll}
\hline Bond & Number & $r_{\text {isolated TCNQ }}(\AA)$ & $r_{\text {adsorbed TCNQ }}(\AA)$ & $\Delta r(\AA)$ \\
\hline $\mathrm{N} \equiv \mathrm{C}$ & 1 & 1.171 & 1.173 & +0.001 \\
$\mathrm{C}-\mathrm{C}$ & 2 & 1.421 & 1.418 & -0.002 \\
$\mathrm{C}=\mathrm{C}$ & 3 & 1.399 & 1.408 & +0.006 \\
$\mathrm{C}-\mathrm{C}$ & 4 & 1.438 & 1.434 & -0.003 \\
$\mathrm{C}=\mathrm{C}$ & 5 & 1.363 & 1.366 & +0.002 \\
\hline
\end{tabular}

Table 3

Bond lengths change for F4-TCNQ before and after adsorption (bonds 1-6 are shown in Fig. 4b).

\begin{tabular}{lllll}
\hline Bond & $\mathrm{Nb}$ & $r_{\text {isolated F4TCNQ }}(\AA)$ & $r_{\text {adsorbed F4TCNQ }}(\AA)$ & $\Delta r(\AA)$ \\
\hline $\mathrm{N} \equiv \mathrm{C}$ & 1 & 1.171 & 1.173 & +0.002 \\
$\mathrm{C}-\mathrm{C}$ & 2 & 1.422 & 1.419 & -0.003 \\
$\mathrm{C}=\mathrm{C}$ & 3 & 1.400 & 1.414 & +0.014 \\
$\mathrm{C}-\mathrm{C}$ & 4 & 1.438 & 1.429 & -0.009 \\
$\mathrm{C}=\mathrm{C}$ & 5 & 1.368 & 1.373 & +0.005 \\
$\mathrm{C}-\mathrm{F}$ & 6 & 1.338 & 1.345 & -0.007 \\
\hline
\end{tabular}

$\sim 0.002$ e from graphene to ANTR molecule was found. This adsorbed system had the smallest binding energy and charge transfer value and biggest binding distance among these organic molecule adsorbed systems. (a)

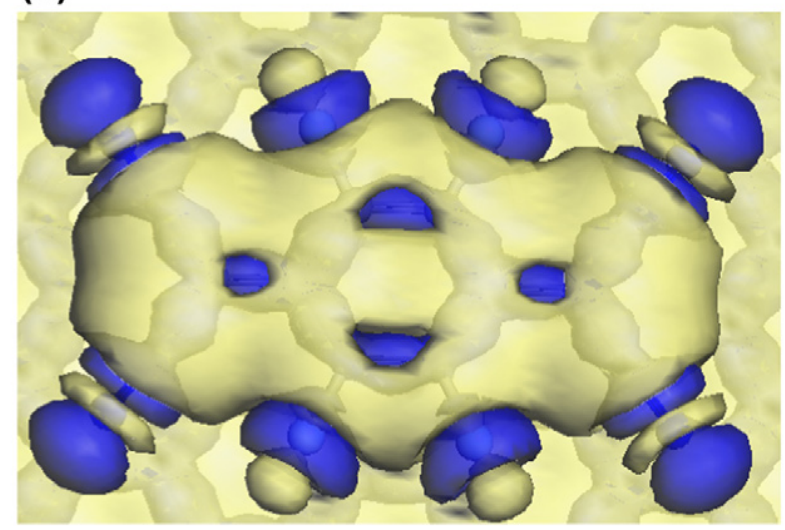

(b)



Fig. 4. Electron density difference isosurfaces (a) (the yellow region shows the electron accumulation, while the blue region shows the electron loss) and a structure representation of the F4-TCNQ molecular (b) (the dark gray, green and blue atoms represent $\mathrm{C}, \mathrm{F}$ and $\mathrm{N}$, respectively) for F4-TCNQ adsorption system.

\subsection{Density of states}

To better understand the bonding properties through electronic structure, the electronic density of states (DOS) of these adsorbed systems were obtained in Fig. 5. In this part, the Kohn-Sham DOS was computed using a $8 \times 8 \times 1 \Gamma$-centered Brillouin-zone sampling, and the energy eigenvalues were smeared with Gaussians of width of $\sigma=0.1 \mathrm{eV}$. Our computation results showed that the pristine graphene was a zero-gap semiconductor, and its Fermi energy exactly crossed the Dirac point, being in agreement with the calculated results of other research [16]. While, the calculated DOS results of graphene in the presence of organic molecules clearly showed discrete molecular levels around the Fermi energy. For TCNE, TCNQ and F4-TCNQ adsorbed systems, the presence of organic molecules resulted to the slightly shifting of Dirac point towards the higher energy level, the electrons were moved from the valence bands of graphene to the TCNE, TCNQ and F4-TCNQ molecules. For TDAE adsorbed systems, the presence of TDAE molecules caused the slightly shifting of Dirac point towards the lower energy level, the electrons were moved from TDAE molecule to the conduction bands of graphene. While for ANTR adsorbed system, there was no significant change for the Dirac point, and this result consisted with our Mulliken population analysis results that almost no charge transfer was found between ANTR and the graphene. Meanwhile, we can explore the hybridized orbitals by analyzing total density of state (TDOS) of adsorbed systems and partial density of state (PDOS) of organic molecules. From Fig. 5 we can see that there was almost no orbital hybridization between organic molecule and the graphene, indicating weak interaction between them. 


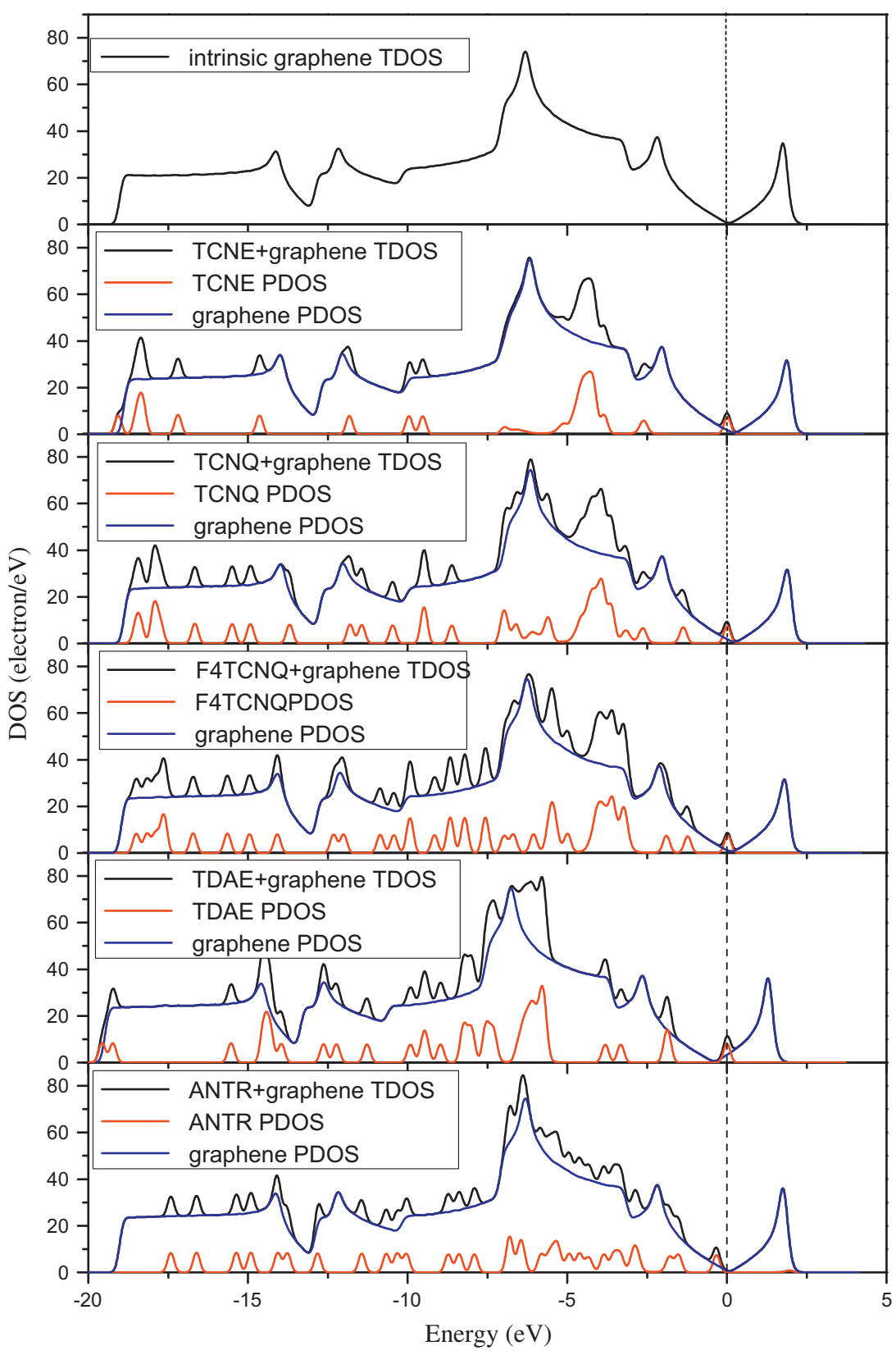

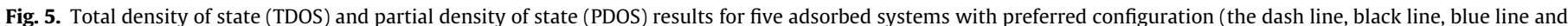
red line indicate the Fermi level, the TDOS of adsorbed systems, the PDOS of graphene and PDOS of organic molecules).

\section{Conclusions}

In summary, with DFT calculations, the adsorptions of the electrophilic molecules (TCNE, TCNQ and F4-TCNQ) and the nucleophilic molecules (TDAE and ANTR) on the graphene sheet was investigated. The computational results showed that the small binding energy and large adsorption distance indicated that organic molecules underwent physical adsorption on graphene surface. Mulliken population analysis results showed that obvious electrons transformation occurred between graphene sheet and TCNE, TCNQ, F4-TCNQ and TDAE, but did not for ANTR. Electrons transferred from the graphene to TCNE, TCNQ and F4-TCNQ, while from TDAE to the graphene sheet. Furthermore, the density of states (DOS) result also was consistent with this. So, by adsorbing different kind of organic molecules, the carrier type and concentration of graphene can be controlled. Our novel results should have great implications for future graphene-based nanoelectronic devices.

\section{Acknowledgements}

This work was jointly supported by National Natural Science Foundation of China (NSFC, Grant No. 60936001, 11072244, 11011120245 and 11021262) and the Instrument Developing Project of the Chinese Academy of Sciences (Grant No. Y2010031).

\section{References}

[1] K.S. Novoselov, A.K. Geim, S.V. Morozov, D. Jiang, Y. Zhang, S.V. Dubonos, I.V. Grigorieva, A.A. Firsov, Science 306 (2004) 666.

[2] F. Schedin, A.K. Geim, S.V. Morozov, E.W. Hill, P. Blake, M.I. Katsnelson, K.S. Novoselov, Nat. Mater. 6 (2007) 652-655. 
[3] C. Berger, Z.M. Song, X.B. Li, X.S. Wu, N. Brown, C. Naud, D. Mayou, T.B. Li, J. Hass, A.N. Marchenkov, E.H. Conrad, P.N. First, W.A. de Heer, Science 312 (2006) 1191-1196.

[4] M.Y. Han, B. Özyilmaz, Y.B. Zhang, P. Kim, Phys. Rev. Lett. 98 (2007) 206805

[5] A. Rycerz, J. Tworzydlo, C.W.J. Beenakker, Nat. Phys. 3 (2007) 172-175.

[6] B. Huard, J.A. Sulpizio, N. Stander, K. Todd, B. Yang, D. Goldhaber-Gordon, Rev. Lett. 98 (2007) 236803.

[7] M. Chi, Y.P. Zhao, Mater. Sci. 46 (2009) 1085-1090

[8] S. Stankovich, D.A. Dikin, G.H.B. Dommett, K.M. Kohlhaas, E.J. Zimney, E.A. Stach, R.D. Piner, S.T. Nguyen, R.S. Ruoff, Graphene-based Compos. Mater. Nat. 442 (2006) 282-286.

[9] J.C. Meyer, A.K. Geim, M.I. Katsnelson, K.S. Novoselov, T.J. Booth, S. Roth, Struct. Suspend. Graphene Shets Nat. 446 (2007) 60-63.

[10] K.S. Novoselov, D. Jiang, F. Schedin, T.J. Booth, V.V. Khotkevich, S.V. Morozov, A.K. Geim, Natl. Acad. Sci. USA 102 (2005) 10451-10453.

[11] K.S. Novoselov, A.K. Geim, S.V. Morozov, D. Jiang, M.I. Katsnelson, I.V. Grigorieva, S.V. Dubonos, A.A. Firsov, Nature 438 (2005) 197-200.

[12] Y. Zhang, J.W. Tan, H.L. Stormer, P. Kim, Nature 438 (2005) 201-204.

[13] K.I. Bolotin, K.J. Sikes, Z. Jiang, M. Klima, G. Fudenberg, J. Hone, P. Kim, H.L. Stormer, Ultrahigh Electron. Mob. Suspend. Graphene Solid State Commun. 146 (2008) 351-355.
[14] S.Y. Zhou, G.-H. Gweon, A.V. Fedorov, P.N. First, W.A. De Heer, D.-H. Lee, F. Guinea, Nat. Mater. 6 (2007) 770-775

[15] W. Chen, S. Chen, D.C. Qi, X.Y. Gao, A.T.S. Wee, Am. Chem. Soc. 129 (2007) 10418-10422

[16] Y.H. Lu, W. Chen, Y.P. Feng, Phys. Chem. B 113 (2009) 2-5.

[17] J. Lu, S. Nagase, D.P. Yu, H.Q. Ye, R.S. Han, Z.X. Gao, S.A. Zhang, L.M. Peng, Rev. Lett. 93 (2004) 116804.

[18] W.Z. Liang, J.L. Yang, J. Sun, Phys. Lett. 86 (2005) 223113.

[19] J.P. Perdew, K. Burke, M. Ernzerhof, Rev. Lett. 77 (1996) 3865-3868.

[20] M. Dolg, W. Liu, S. Kalvoda, Quant. Chem. 76 (2000) 359-370.

[21] R. Fletcher, Practical Methods of Optimization, vol. 1, Wiley, New York, 1980

22] R.G.A. Veiga, R.H. Miwa, Rev. B 73 (2006) 245422.

[23] T.J. Emge, M. Maxfield, D.O. Cowan, T.J. Kistenmacher, Mol. Cryst. Liq. Cryst. 65 (1981) $161-178$

[24] G.M. Rangger, O.T. Hofmann, L. Romaner, G. Heimel, B. Bröker, R. Blum, R.L. Johnson, N. Koch, E. Zojer, Rev. B 79 (2009) 165306.

[25] R. Saito, G. Dresselhaus, M.S. Dresselhaus, Imperial College, London, UK, 1998. 